\title{
Location and Number of Nutrient Foramina in Human Long Bones of a Sri Lankan sample
}

\author{
Subasinghe SAJS ${ }^{1}$, Weerasooriya WWRWMPK ${ }^{1}$, Fernando RCU ${ }^{1}$, Ekanayake EMSD', \\ Jayaweera $\mathrm{AS}^{1}$, Nawarathna $\mathrm{NGS}^{1}$, Sampath $\mathrm{PH}^{1}$, Manawarathne MRK ${ }^{1}$, Banneheka \\ BMHSK $^{1}$, Peiris HRD ${ }^{1}$ \\ ${ }^{\prime}$ Division of Anatomy, Department of Basic Sciences, Faculty of Dental Sciences, \\ University of Peradeniya. \\ *susansubasinghe@gmail.com
}

Nutrient foramen is an opening in the shaft of the bone from which nutrient arteries enter the medullary cavity providing the main blood supply for long bones. Although foramina in long bones are located away from the growing end, the topographical anatomy may vary and can even alter during growth. The objective of the present study was to determine the location and the number of the nutrient foramina in long bones of a Sri Lankan population. In this study 253 adult human long bones of the upper (43 humeri, 43 radii, 48 ulnae) and lower (52 femora, 49 tibiae, 38 fibulae) limbs were used. For each bone the foraminal index indicating the location of the nutrient foramen in relation to its proximal end was calculated (Foraminal index=Distance of the foramen from the proximal end/Total length). In the upper limb, foramina were located on the diaphysis with foraminal index ranging from 5.2 $73.84 \%$ of the overall length of the humerus, $11.11-55.16 \%$ for the radius and $9.34-73.64 \%$ for the ulna. In the lower limb foramina were located on the diaphysis $3.97-89.74 \%$ of the overall length of the femur, 11.76$72.17 \%$ for the tibia and $23.42-56.76 \%$ for the fibula. In addition, the number and the distribution of the foramina in relation to specific regions/surfaces of the diaphysis were identified. This study provides additional and important information on the location and number of nutrient foramina in the long bones of the upper and lower limbs in a Sri Lankan sample. The knowledge of these variations is important during surgical procedures in order to preserve nutrient arteries. 